\title{
Functional Outcomes in Spinal Tuberculosis: A Review of the Literature
}

\author{
Lionelson Norbert Yong ${ }^{1}$, Fatimah Ahmedy ${ }^{1}$, Khin Nyein Yin ${ }^{1}$, Julia Patrick Engkasan ${ }^{2}$ \\ ${ }^{1}$ Faculty of Medicine \& Health Sciences, Universiti Malaysia Sabah, Kota Kinabalu, Malaysia \\ ${ }^{2}$ Department of Rehabilitation Medicine, Faculty of Medicine, University of Malaya, Kuala Lumpur, Malaysia
}

\begin{abstract}
Spinal tuberculosis often leads to neurological deficit and subsequent deterioration in functional outcomes. This review assesses the recent evidence on functional outcomes in spinal tuberculosis, highlighting functional recovery, assessment tools for functional measures, and associative factors for functional recovery. Using PubMed, a literature search was done using the terms "spinal tuberculosis," "tuberculous spondylitis," "tuberculous spondylodiscitis," and "functional outcome" for original articles published between January 2010 and December 2019. A total of 191 search results were found. Detailed screening showed that 19 articles met the eligibility criteria: 13 of these focused on surgical methods, four on conservative management, and two on rehabilitation approaches. The outcome measures used for functional assessment were the Oswestry Disability Index (11 articles), Japanese Orthopaedic Association score ( $n=3)$, modified Barthel Index ( $n=2)$, Functional Independence Measure ( $n=2)$, and 36-item Short-Form Health Survey ( $n=1)$. Functional outcome was mainly affected by pain, spinal cord compression, and inpatient rehabilitation. No significant difference in functional outcome was found between conservative management and surgery for cases with uncomplicated spinal tuberculosis. Most studies focused on surgery as the mode of treatment and used pain-related functional measures; however, these assessed functional limitations secondary to pain, and not neurological deficits. Further studies may consider examining functional outcomes in spinal tuberculosis by utilizing spinal cord-specific functional outcome measures, to evaluate outcome measures as a prognostic tool, and to measure functional outcomes from specific rehabilitation interventions.
\end{abstract}

Keywords: Spinal tuberculosis; Outcome measures; Review; Spinal cord disorders

\section{Introduction}

In 2017, an estimated 10 million people reported tuberculous infections worldwide, with Southeast Asia seen to have the second-highest prevalence, at 226 cases per 100,000 population [1]. Spinal tuberculosis is the most common subtype of extra-pulmonary tuberculosis, accounting for $15 \%$ of cases [2]. The infection's proximity to the spinal cord and nerves often leads to neurological symptoms such as pain, weakness, and numbness in the lower limbs. If left untreated, spinal tuberculosis may go on to cause serious morbidity, residual neurological deficits, and severe deformities. All of this contributes to the loss of functional abilities, especially in terms of mobility, leading to difficulty in walking, self-care, and management of the sphincter. This in turn can bring about further physical, mental, emotional, and social deterioration, and adversely affect the patient's quality of life.

Received Feb 26, 2020; Revised May 11, 2020; Accepted Jun 9, 2020

Corresponding author: Fatimah Ahmedy

Faculty of Medicine \& Health Sciences, Universiti Malaysia Sabah, Jalan UMS, 88400 Kota Kinabalu, Sabah, Malaysia

Tel: +60-13-8805513, Fax: +60-88320928, E-mail: fatimahmedy@ums.edu.my 
Patients with spinal tuberculosis should undergo rehabilitation to achieve the best functional outcome based on their neurological deficit [3]. This always involves prognostication that guides therapeutic interventions and is heavily influenced by functional outcome. The established literature for spinal tuberculosis mostly comprises original studies, while review articles are readily available and are primarily concerned with diagnostic investigation and intervention to improve pain and deformity, rather than specifically emphasizing functional outcomes such as walking and other daily activities [4-6]. However, up-todate reviews on functional outcomes are still lacking.

We aim to systematically organize and review the established evidence on functional outcomes in spinal tuberculosis, highlighting functional recovery, assessment tools for functional measures, and associative factors for functional recovery. The outcomes from this review should enhance clinicians' understanding of functional outcomes and prognostication when treating individuals with spinal tuberculosis.

\section{Methods}

\section{Search methodology}

Studies published between January 2010 and November 2019 have been included in this review, which was carried out in December 2019 using Medline's large database of good quality studies. We decided on this as our sole search database as a means to ensure that all the selected studies had gone through standardized publication protocols. Two researchers searched for the terms "spinal tuberculosis" OR "tuberculous spondylitis" OR "tuberculous spondylodiscitis" AND "functional outcome" and the results were screened based on various inclusion and exclusion criteria. Additional manual searches were conducted based on the references contained in the selected articles. A third reviewer was invited to resolve disagreements when necessary.

\section{Study selection}

The selected articles were all published in English. We considered studies that determined functional assessment among the outcomes in adults (age 18 years and above) diagnosed with spinal tuberculosis. Case reports, review articles, technical reports, and thesis dissertations were excluded, as were abstract-only publications. Studies that included pyogenic spinal infection were also excluded due to the typical feature of single-level vertebral involvement with better neurological and functional outcomes compared to spinal infection with tuberculosis etiology. Studies that used subjective outcome measures such as the Prolo Scale and modified MacNab criteria were also not included in this review.

\section{Data extraction and recording}

The following data were extracted and recorded: (1) details of each article (title, author, year of publication, study design, sample size, and mean follow-up duration); (2) demographic and clinical characteristics of the studied population (mean age, male/female ratio, and neurological status); and (3) study outcomes (researched variables, outcome measures of functional assessment, and corresponding results). Ethical approval was not required since the review was based on previously published articles.

\section{Results}

Our search yielded 191 results. After a detailed screening of these articles based on the study selection criteria, 17 papers were chosen. A further two relevant studies extracted from the reference lists were manually added, bringing the total to 19 articles. Fig. 1 illustrates the selection process through a flowchart, while the information gathered is tabulated in Table 1.

\section{Modes of treatment}

The selected articles emphasized the varying modes of treatment. A total of 13 articles focused on surgical methods, four were on conservative management, and two explored rehabilitation.

Surgery via the posterior approach was the most customary studied surgical method among the selected articles. Singh et al. [2], Sun et al. [7], and Ran et al. [8] found that the posterior spinal surgical approach produced a significant improvement in the AIS score among patients with AIS B, AIS C, and AIS D on admission, but lesser improvement was observed in those with AIS A. However, Omran and Abdel-Fattah [9] found that the posterior extensive circumferential decompressive reconstructive technique could also improve the neurological outcome 
191 Potential studies of interest

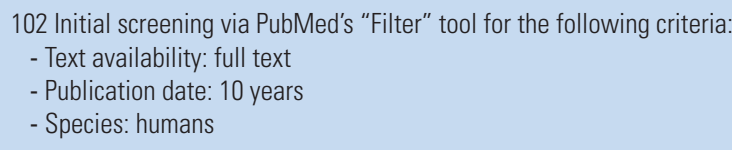

17 Manual evaluation of abstracts to exclude:

- Case reports, review articles, and technical reports

- Articles that do not study spinal tuberculosis or patient's functional outcome

2 Manually searched articles from the reference list of the selected articles that are relevant to the study

19 Total selected studies

Fig. 1. Flowchart demonstrating the selection process of eligible articles for review.

of patients who were initially graded as AIS A. Posterior spinal stabilization surgery with titanium mesh or autogenous iliac bone graft also demonstrated improvement in the Frankel Grading, from grade D to E [10]. Meanwhile, surgery via the anterior approach was shown to improve the level of disability based on Japanese Orthopaedic Association (JOA) scores among patients with cervical spinal tuberculosis, as shown in studies by Xing et al. [11] and Liu et al. [12].

Comparative studies examining neurological recovery between anterior and posterior approaches for those with thoracic level spinal tuberculosis did not reveal significant differences in the AIS score [13,14]. Recent technology improvements have allowed minimally invasive surgery to be used as a surgical stabilization technique for spinal tuberculosis via video-assisted thoracoscopic surgery (VATS), and improved those with Frankel grade A to grade D or E [15].

All of the studies used anti-tuberculous chemotherapy as their primary mode of treatment in the conservative management of spinal tuberculosis. In this way, patients were given isoniazid, rifampicin, pyrazinamide, and ethambutol for 2 months, followed by isoniazid and rifampicin only for the following 16 months [16-19]. Meanwhile, Shetty et al. [18] also mentioned the prescription of 2-3 weeks bed rest followed by in-house ambulation and lumbosacral bracing for patients who needed it. In Qu et al. [19], concurrent percutaneous needle aspiration was also done to drain the tuberculous abscess.

Of the four studies that researched conservatively managed spinal tuberculosis patients, two evaluated the factors influencing the outcome, which will be elaborated on later, and the remaining two compared the outcomes between surgical approach and conservative management. Shetty et al. [18] showed an inverse relationship between the presence of lumbar lordosis and Oswestry Disability Index (ODI). At the same time, Qu et al. [19] found that in uncomplicated spinal tuberculosis, there was no significant difference in the functional outcome of patients who were conservatively managed, as opposed to those who underwent surgery.

Regardless of the mode of initial treatment, all the spinal tuberculosis patients in the chosen studies eventually received rehabilitation management, and Zaoui et al. [20] found that the mean of modified Barthel Index (MBI) significantly increased from 75.5 to 92.65 with inpatient rehabilitation after 6 months of diagnosis. Zaoui et al. [20] stressed the need for postoperative immobilization with the use of orthoses, either in the form of a corset or cervical collar, when rehabilitation was initiated. This was followed by early and progressive verticalization by physiotherapy that aimed for the prevention of decubitus complications while preserving articular, muscular, trophic, and respiratory functions. Muscle strengthening of the limbs was conducted with axial resistance exercises in adjunct with postural training for balance. The use of wheelchairs, walking frames, and crutches were beneficial for assisting mobilization. The rehabilitation training given should be adapted to the individualized functional recovery levels of the patients [20]. Although Zaoui et al. [20] found significant neurological improvement through rehabilitation only in patients with AIS B to E classification, Toulgui et al. [21] found substantial neurological recovery even in those with complete paraplegia (AIS A).

\section{Functional outcome assessment}

The selected articles used a variety of tools to assess functional outcome, as displayed in Table 1. These included ODI, JOA, MBI, Functional Independence Measure (FIM), and 36-item Short-Form Health Survey (SF-36).

Each of these scoring systems covers different domains 


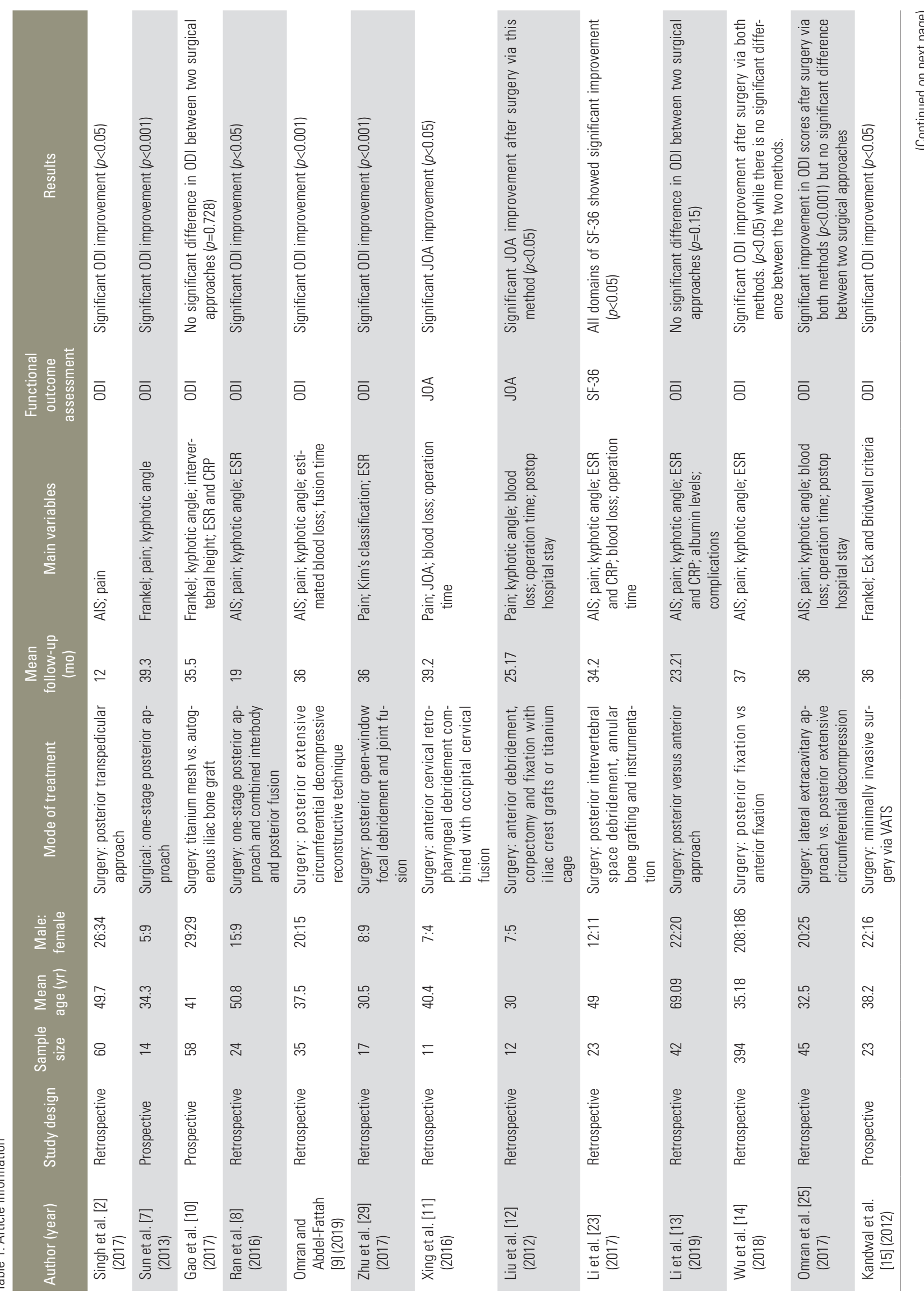




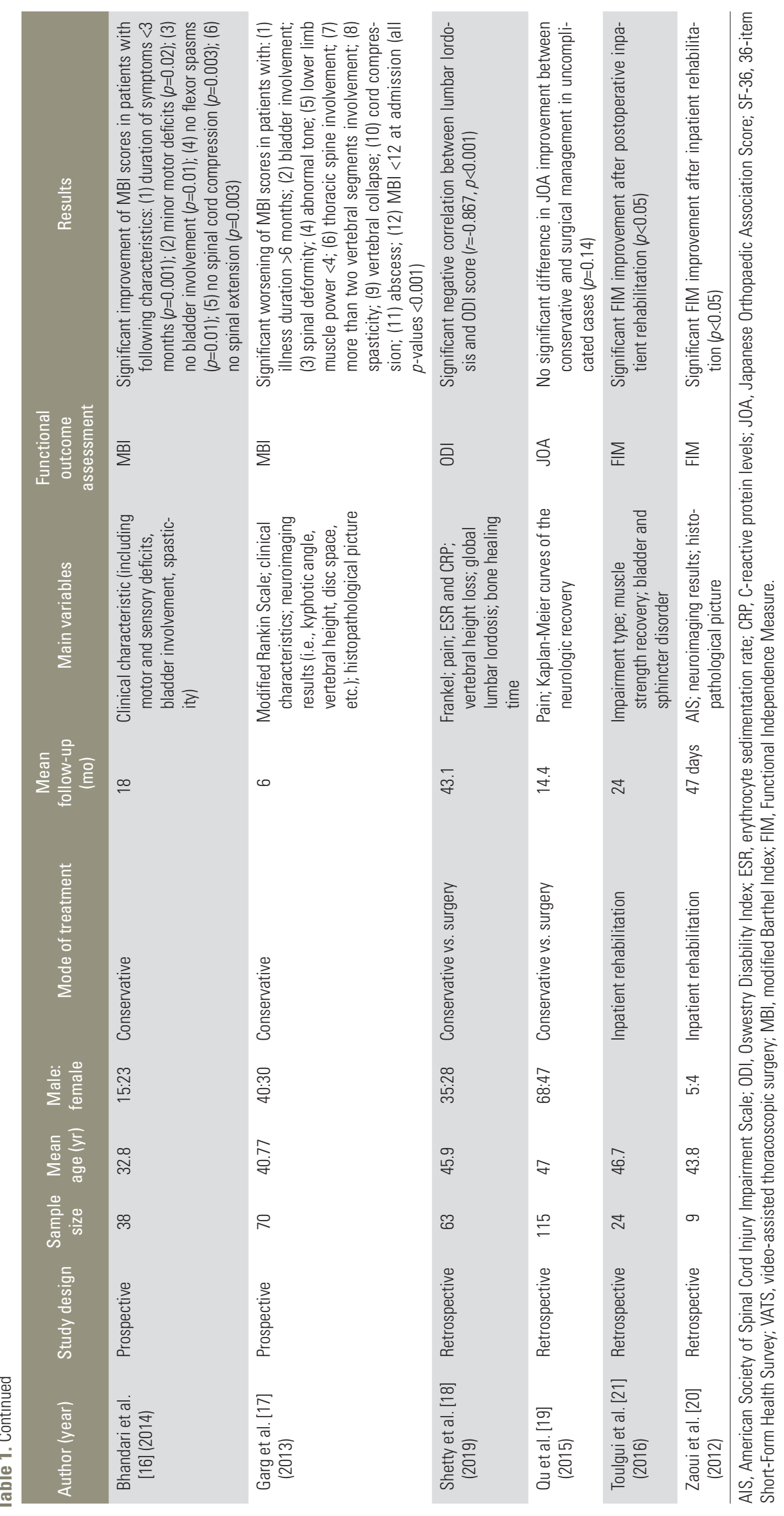


of functional outcome. For instance, ODI assesses functional disability secondary to low back pain. The JOA scores the patient's pain, neurological function, and basic daily living activities. Meanwhile, MBI and FIM both focus on the functional limitations from the resulting neurological deficits, such as self-care, sphincter control, respiration, and mobility, with a higher degree of sensitivity. SF-36 is directed more toward the effects of the neurological deficits on social functioning, whereby it assesses the impact of the illness on patients' physical activities, general health, occupational issues, and mental health.

ODI is used in a majority of the selected articles (11 out of 19). It was noted that studies focusing on the outcomes from surgical intervention mostly utilize ODI or JOA to measure function. In contrast, studies examining the functional outcome from either conservative management or rehabilitation used MBI and FIM.

A total of 10 of these articles assessed the severity of pain using a Visual Analog Scale (VAS) as one of the main outcomes, with all showing a mean VAS score higher than 5. This suggests that pain is a major disturbing symptom experienced by spinal tuberculosis patients and is very likely to have an impact on their functional outcome.

\section{Functional recovery}

\section{1) Conservative management}

The functional recovery of patients under conservative management was studied by Bhandari et al. [16] and Garg et al. [17]. Both Bhandari et al. [16] $(n=38)$ and Garg et al. [17] ( $\mathrm{n}=70)$ applied the same conservative management by using anti-tubercular chemotherapy for 18 months with adequate pain management. Both studies also had similar mean age (32.84 \pm 20.52 years and $40.77 \pm 15.12$ years, respectively) and percentage of patients with spinal cord compression (52.6\% and 58.6\%, respectively). However, they differed in the percentage of patients with baseline motor deficits ( $47.4 \%$ and $90 \%$, respectively) and baseline MBI scores of less than 12 (76.3\% and 52.9\%) $[16,17]$.

Garg et al. [17] reported that conservatively managed patients with certain clinical characteristics were found to have significantly lower MBI scores. These characteristics included symptoms for more than 6 months prior to spinal tuberculosis diagnosis, neurogenic bladder complication, spinal deformity, abnormal muscle tone, lower limb muscle strength of less than 4 based on Medical Research
Council grading, thoracic level, multiple vertebral segment involvement, significant spasticity, vertebral collapse, spinal cord compression, the formation of abscess, and an MBI score of $<12$ on admission $(p<0.001)$. On the other hand, Bhandari et al. [16] reported a total of $81.6 \%$ of conservatively managed spinal tuberculosis patients had witnessed improvements in their MBI score. They found that those who had endured symptoms for less than 3 months before spinal tuberculosis diagnosis and with a milder form of motor deficit had significantly higher MBI improvements ( $p=0.001$ and $p=0.02$, respectively) [16]. This implies that early diagnosis and prevention of spinal cord-related consequences are vital components for improving functional outcome.

\section{2) Surgical treatment}

In complicated spinal tuberculosis cases, such as those with the presence of spinal cord compression secondary to extensive abscess, surgical stabilization surgery is highly emphasized [22]. Surgical stabilization decompresses the spinal cord, strengthens the structure of the damaged spine, reduces pain, and corrects the resultant deformity. As a result, this surgical method may reduce the risk of further damage to the spinal cord while also allowing stability for postoperative rehabilitation training. The basic principle of surgery is to perform surgical decompression by intervertebral debridement, followed by vertebral fixation. The method of fixation may be done with autologous bone grafts or via a titanium cage, both of which showed excellent functional outcome in terms of JOA [12]. Based on the findings of Ran et al. [8], Li et al. [23], and Wu et al. [14], the decision to use the anterior or posterior approach in surgery should be determined in individual cases based on the infected segments, the severity of deformity, and the presence of cord compression. However, the use of minimally invasive surgery such as VATS is seen to be a good "middle-ground" between the surgical and conservative management of spinal tuberculosis patients as it was found to significantly improve ODI score [22].

\section{3) Rehabilitation approach}

For both surgical and conservative management, inpatient rehabilitation is necessary as it is shown to improve FIM $[21,20]$. Toulgui et al. [21] emphasize the importance of initial mobilization whereby initial mobilization of the injured segment is recommended with the aid of orthosis. The basic principle of rehabilitating individuals with spi- 
nal tuberculosis involves prescribing isometric exercises during the early phase of treatment, followed by strengthening of spinal extensor and isotonic exercises of the abdomen, pelvis and lower limbs (the latter for incomplete motor paraparesis) [24]. Gradual retraining of the mobility function and performance of daily living activities improve the functional outcome. The study by Toulgui et al. [21] showed that the average FIM of patients who received inpatient rehabilitation improved from 62.3 to 112 at 2 years after diagnosis (mean inpatient rehabilitation stay of 32 days) followed by regular outpatient rehabilitation follow-up. There is insufficient literature to compare the functional recovery in spinal tuberculosis patients who had rehabilitation and those who did not.

\section{4) Pain reduction}

Significant functional recovery was found in all cases that recorded reduced pain score (Table 2). The functional outcome measures used were ODI, JOA, and SF-36; in which the former two primarily evaluate the performances of daily activities concerning pain in the back.

\section{Discussion}

This review revealed that surgical intervention is more commonly used than conservative management. This is probably due to the neurological consequences leading to disruption of functional abilities, emphasizing the indication for surgery. Studies that examined surgery techniques recorded a considerable number of patients with compromised preoperative neurological status [2,7$10,13-15,23,25]$. On the other hand, fewer studies evaluated functional outcomes from conservative management among individuals with spinal tuberculosis; however, those who underwent conservative management were less likely to have neurological deficits to begin with $[16,17]$. Our literature search failed to identify articles that studied the functional outcomes of specific non-surgical methods for stabilizing spinal tuberculosis, such as thoracolumbar orthosis. Nevertheless, these selected studies described in detail the resultant functional outcomes with the clinical characteristics.

There is still insufficient evidence to determine functional outcomes for spinal tuberculosis. Rehabilitation practitioners therefore have to rely on the knowledge and understanding of the prognostication of rehabilitating other etiologies of non-traumatic spinal cord injury as a means of predicting the outcomes from spinal tuberculosis. For instance, Vervoordeldonk et al. [26] found that patients with infective etiology of spinal cord injury did see functional recovery from inpatient rehabilitation intervention within an average of 83 days. However, this study did not compare in detail the different types of infective etiology. Hence, the results may vary for spinal tuberculosis.

As discussed earlier, ODI and JOA are commonly used functional outcome assessments. However, ODI has limitations, whereby it only assesses functional disability secondary to low back pain and is used for various low back conditions, including degenerative etiologies $[27,28]$.

Table 2. VAS scores and functional outcome

\begin{tabular}{|c|c|c|c|}
\hline \multirow{2}{*}{ Author (year) } & \multicolumn{2}{|c|}{ Mean pain score (VAS) } & \multirow{2}{*}{ Post-intervention functional outcome at last FU } \\
\hline & Pre-treatment & At last FU & \\
\hline Singh et al. [2] (2017) & 8.15 & 0.83 & Significant ODI improvement $(p<0.05)$ \\
\hline Sun et al. [7] (2013) & 7.43 & 0.5 & Significant ODI improvement $(p<0.05)$ \\
\hline Gao et al. [10] (2017) & $5.7-5.9$ & $1.1-1.8$ & Significant ODI improvement $(p<0.05)$ \\
\hline Omran and Abdel-Fattah [9] (2019) & 7.47 & 1.21 & Significant ODI improvement $(p<0.05)$ \\
\hline Zhu et al. [29] (2017) & 6.0 & 0.5 & Significant ODI improvement $(p<0.05)$ \\
\hline Li et al. [13] (2019) & $6.3-7.3$ & $1.8-2.0$ & Significant ODI improvement $(p<0.05)$ \\
\hline Omran et al. [25] (2017) & $7.28-7.43$ & $1.00-1.21$ & Significant ODI improvement $(p<0.05)$ \\
\hline Qu et al. [19] (2015) & $4.8-5.6$ & 0 & Significant ODI improvement $(p<0.05)$ \\
\hline Xing et al. [11] (2016) & 6.73 & 0.64 & Significant JOA score improvement $(p<0.05)$ \\
\hline Li et al. [23] (2017) & 8.1 & 1.2 & Improved SF-36 score $(p<0.05)$ \\
\hline
\end{tabular}

VAS, Visual Analog Scale; FU, follow-up; ODI, Oswestry Disability Index; JOA, Japanese Orthopaedic Association; SF-36, 36-item Short-Form Health Survey. 
Although the use of ODI and JOA is related to the likelihood of developing pain in spinal tuberculosis, they do not truly reflect the functional limitations caused by the neurological deficits. Both of these outcome measures focus on the pain affecting mobility and daily functions. Hence it would be misleading for individuals with nil or insignificant pain to answer appropriately because a majority of functional disabilities from spinal tuberculosis are secondary to lower limb weakness or sensory loss.

The use of MBI, FIM, and SF-36 provide a more holistic perspective. The former two focus on the functional limitations of the neurological deficits while SF-36 emphasizes social functioning and quality of life. Nevertheless, both MBI and FIM have been validated as outcome measurement tools and applied in major neurological disorders, including stroke and traumatic brain injury. Although these provide objective assessments with good inter-rater reliability, the tools do not assess in detail the extent of functional disability commonly associated with spinal cord-related disorders such as spinal tuberculosis. On the other hand, SF-36 assesses very few functional limitations, as this tool is primarily concerned with social participation and quality of life.

Thus far, we have not found studies that used spinal a cord-specific functional assessment tool such as the Spinal Cord Independence Measure-III (SCIM-III). This outcome measure is designed to assess patients with spinal cord-related disorders and has excellent reliability (internal consistency of $>0.84$ ) and validity $(r=0.92)$ [28]. It would be worthwhile to consider SCIM-III as a specific measure for functional outcomes and a prognostication tool for future studies evaluating certain treatment and rehabilitation interventions for spinal tuberculosis.

Three major factors were found to improve functional recovery in spinal tuberculosis patients: pain reduction, decompression of the spinal cord, and inpatient rehabilitation. Patients with spinal tuberculosis who had less pain were more likely to voluntarily perform daily living activities and rehabilitative exercises, whereas those with insufficient pain reduction were likely to be more reticent in performing these activities.

Spinal cord compression is a major factor that affects functional outcome in patients with spinal tuberculosis. Among the clinical characteristics found to be associated with poor functional recovery were symptoms of spinal cord compression (neurogenic bowel and bladder, muscle spasms, and weakness) and the cause of the cord com- pression itself (spinal deformity, thoracic spine involvement, vertebral collapse, abscess formation, and severe lordosis) [16-18]. Consequently, patients who underwent spinal decompression and stabilization surgery showed significant improvement in functional outcome, as shown in Table 1. Although various surgical techniques feature among our selected articles, all involved spinal debridement, osteotomy, and stabilization, whether by grafting or instrumentation. These techniques decompress the spinal cord to relieve the impingement and fixate the spine to prevent further injury $[2,7-14,23,25,29]$. In short, spinal cord compression requires surgical intervention to promote neurological recovery and permits early initiation of rehabilitative training for improvement in functional outcomes.

An alternative to open spinal cord decompression is the use of minimally invasive surgery such as VATS. A study by Kandwal et al. [15] compared the outcome of VATS and mini-open posterolateral decompression in spinal tuberculosis patients. Both groups of patients had a similar mean age ( 38.2 years and 32.5 years, respectively) and preoperative kyphotic angles ( $38^{\circ}$ and $41.25^{\circ}$, respectively). Both methods showed equally significant ODI recovery $(p<0.05$ in both groups). Although VATS was mainly performed in patients with single-level, thoracic spinal tuberculosis, compared to the open surgery conducted in those with multi-level thoracolumbar infections, VATS was proven to produce significant functional, structural, and neurological recovery with significantly less blood loss and postoperative morbidity.

The effects of spinal cord compression on functional outcome may also indirectly skew the data, giving the impression that patients who undergo surgical management as the main treatment for spinal cord compression have poorer functional outcome. Conversely, patients who do not have spinal cord compression, and thus undergo conservative management, have better functional outcomes. This leads to a possibly false assumption that the type of intervention may affect functional recovery. In practice, the suitability of the type of management on an individual case may influence the outcome on the functional recovery.

For both conservative and surgical management, rehabilitation is essential in a patient's functional recovery. Rehabilitation involves retraining spinal tuberculosis patients in their performance with functional activities. In terms of rehabilitation, the main goal in treating spinal 
tuberculosis is to achieve recovery through adequate mobilization, as immobilization could lead to complications such as contractures, pressure ulcers, and muscle wasting. The rehabilitation goals for spinal tuberculosis are specified at each of the spinal tuberculosis stages (acute, subacute, and chronic) [30]. In the acute stage, the goal is to achieve optimal bed mobilization; for example, isometric exercises to strengthen the paraspinal and gluteal muscles are essential for standing, balance, and ambulation exercises in later stages [30]. The subacute stage focuses on active and active-assisted exercises followed by retraining of sitting balance and standing with or without assistance or aids [30]. Ambulation is primarily used at the chronic stage, through a gradual increase in the intensity in the gait retraining and abdominal core exercises [30].

Patients rehabilitating from spinal tuberculosis share similar features to those with other spinal cord-related disorders, such as spasticity, neuropathic pain, and neurogenic bowel and bladder. Spasticity is usually managed with anti-spasmodic drugs such as baclofen and botulinum neurotoxin, while gabapentin is a commonly used analgesic to treat neuropathic pain [31]. Neurogenic bladder and bowel are common consequences of spinal tuberculosis that can be socially debilitating, thus requiring special attention through rehabilitation. A bowel control regimen consisting of timely fecal evacuation, dietary modification and supplementation, including high-fiber diet, adequate water intake, and oral laxatives such as lactulose, is used to treat neurogenic bowel. Fecal evacuation is achieved via bisacodyl suppository and digital stimulation (manual evacuation may also be done, but not as often). The use of bisacodyl suppositories and oral laxatives, an evacuation frequency less than daily, and water intake of more than $2 \mathrm{~L}$ daily were significantly associated with a longer duration for bowel regime intervals and lesser occurrence of accidental bowel output ( $p=0.001,0.001,0.024$, and 0.038 , respectively) [32]. Conservative methods used to improve bladder drainage include 4-6 hourly clean intermittent catheterization, or indwelling urinary catheter if this is not possible, with anticholinergic treatment to enhance bladder capacity. Surgical approaches such as cystoplasty, artificial urinary sphincter and abdominal slings are used only if the initial methods have been exhausted, with deterioration in renal function [33].

Most rehabilitation regimes are best outlined to the patient during inpatient rehabilitation. Both studies examining the effect of inpatient rehabilitation on functional outcomes have demonstrated significant improvement in FIM [20,21]. Regardless whether patients were treated conservatively or surgically in the initial treatment for spinal tuberculosis, neurologically impaired individuals need to undergo retraining and mobilization of their muscles. Preserving muscle bulk and strength prevent deconditioning, which would otherwise be expected due to ongoing weakness from the disease course. Doing so also promotes neurological recovery and psychological wellness. An inpatient rehabilitation facility offers the additional benefit of providing comprehensive and supervised physical and occupational therapy with individualized training.

However, when studying the association between rehabilitation therapy and the functional recovery of spinal tuberculosis patients, it is crucial to recognize the severity of infection, and the management type as a possible confounding factor. The risk of this happening can be reduced by performing an intragroup study in patients at each mode of treatment. Nas et al. [24] have demonstrated that among conservatively managed patients there was significant functional improvement in those who had rehabilitation. This result was similar to those who were surgically managed, illustrating that rehabilitation does improve the rate of functional recovery regardless of the initial mode of treatment.

Despite the fact that functional outcomes are the core feature of rehabilitation medicine, no studies have evaluated how the initial functional outcome measured in spinal tuberculosis can be used as a prognostic indicator for functional improvement after a period of time. Furthermore, other factors related to rehabilitation, such as the intensity of exercises, types of regime, and outcome measure tools on the functional outcome of individuals with spinal tuberculosis, have yet to be determined. While we found some evidence of spinal tuberculosis management and functional recovery in our review, these are still areas that are worth investigating for future studies.

There have been limited studies on spinal tuberculosis and functional outcomes within the past ten years despite the high prevalence of this disease, especially in developing regions. The studies selected for this review mainly concern surgical management and the predominant use of ODI and JOA as measures of functional outcome. The literature search was conducted using a single database. 


\section{Conclusions}

Surgery remains the preferred mode of treatment, especially for managing complicated spinal tuberculosis. Functional outcomes among those with spinal tuberculosis are affected by pain, spinal cord compression, and rehabilitative intervention. Future works on exploring the functional outcomes of patients with spinal tuberculosis may wish to focus on utilizing an outcome measure that is more specific to spinal cord-related disorders, considering initial functional outcome as a prognostic tool, and measuring the effectiveness of specific rehabilitative regimens in the functional outcome.

\section{Conflict of Interest}

No potential conflict of interest relevant to this article was reported.

\section{Acknowledgments}

This work has been supported by the Universiti Malaysia Sabah's Research Grant.

\section{Author Contributions}

Lionelson Norbert Yong: first reviewer and main author; Fatimah Ahmedy: second reviewer and corresponding author; Khin Nyein Yin: third reviewer; and Julia Patrick Engkasan: review the overall write-up.

\section{References}

1. MacNeil A, Glaziou P, Sismanidis C, Maloney S, Floyd K. Global epidemiology of tuberculosis and progress toward achieving global targets: 2017. MMWR Morb Mortal Wkly Rep 2019;68:263-6.

2. Singh S, Dawar H, Das K, Mohapatra B, Prasad S. Functional and radiological outcomes of anterior decompression and posterior stabilization via posterior transpedicular approach in thoracic and thoracolumbar Pott's disease: a retrospective study. Asian Spine J 2017;11:618-26.

3. Yen HL, Kong KH, Chan W. Infectious disease of the spine: outcome of rehabilitation. Spinal Cord 1998;36:507-13.

4. Ali A, Musbahi O, White VLC, Montgomery AS.
Spinal tuberculosis: a literature review. JBJS Rev 2019;7:e9.

5. Garg RK, Somvanshi DS. Spinal tuberculosis: a review. J Spinal Cord Med 2011;34:440-54.

6. Trecarichi EM, Di Meco E, Mazzotta V, Fantoni M. Tuberculous spondylodiscitis: epidemiology, clinical features, treatment, and outcome. Eur Rev Med Pharmacol Sci 2012;16 Suppl 2:58-72.

7. Sun L, Song Y, Liu L, Gong Q, Zhou C. One-stage posterior surgical treatment for lumbosacral tuberculosis with major vertebral body loss and kyphosis. Orthopedics 2013;36:e1082-90.

8. Ran B, Xie YL, Yan L, Cai L. One-stage surgical treatment for thoracic and lumbar Spinal tuberculosis by transpedicular fixation, debridement, and combined interbody and posterior fusion via a posterior-only approach. J Huazhong Univ Sci Technolog Med Sci 2016;36:541-7.

9. Omran K, Abdel-Fattah AS. Posterior extensive circumferential decompressive reconstructive technique in surgical treatment of upper thoracic spine compressive lesions. World Neurosurg 2019;123:e501-8.

10. Gao Y, Ou Y, Deng Q, He B, Du X, Li J. Comparison between titanium mesh and autogenous iliac bone graft to restore vertebral height through posterior approach for the treatment of thoracic and lumbar spinal tuberculosis. PLoS One 2017;12:e0175567.

11. Xing S, Gao Y, Gao K, Zhang G, Yu Z. Anterior cervical retropharyngeal debridement combined with occipital cervical fusion to upper cervical tuberculosis. Spine (Phila Pa 1976) 2016;41:104-10.

12. Liu Y, Chen Y, Yang L, et al. The surgical treatment and related management for post-tubercular kyphotic deformity of the cervical spine or the cervicothoracic spine. Int Orthop 2012;36:367-72.

13. Li Z, Lei F, Xiu P, et al. Surgical management for middle or lower thoracic spinal tuberculosis (T5-T12) in elderly patients: posterior versus anterior approach. J Orthop Sci 2019;24:68-74.

14. Wu W, Lyu J, Liu X, et al. Surgical treatment of thoracic spinal tuberculosis: a multicenter retrospective study. World Neurosurg 2018;110:e842-50.

15. Kandwal P, Garg B, Upendra B, Chowdhury B, Jayaswal A. Outcome of minimally invasive surgery in the management of tuberculous spondylitis. Indian J Orthop 2012;46:159-64.

16. Bhandari A, Garg RK, Malhotra HS, et al. Outcome 
assessment in conservatively managed patients with cervical spine tuberculosis. Spinal Cord 2014;52:48993.

17. Garg RK, Raut T, Malhotra HS, et al. Evaluation of prognostic factors in medically treated patients of spinal tuberculosis. Rheumatol Int 2013;33:3009-15.

18. Shetty AP, Bosco A, Rajasekaran S, Kanna RM. Does preserving or restoring lumbar lordosis have an impact on functional outcomes in tuberculosis of the lumbosacral region? Spine Deform 2019;7:356-63.

19. Qu JT, Jiang YQ, Xu GH, et al. Clinical characteristics and neurologic recovery of patients with cervical spinal tuberculosis: should conservative treatment be preferred?: a retrospective follow-up study of 115 cases. World Neurosurg 2015;83(5):700-7.

20. Zaoui A, Kanoun S, Boughamoura H, et al. Patients with complicated Pott's disease: management in a rehabilitation department and functional prognosis. Ann Phys Rehabil Med 2012;55:190-200.

21. Toulgui E, Jemni S, Frioui S, Lazreg N, Mtaouaa S, Khachnaoui F. Management in a rehabilitation department in Pott's disease. Ann Phys Rehabil Med 2016;59:e96.

22. Kandwal P, G V, Jayaswal A. Management of tuberculous infection of the spine. Asian Spine J 2016;10:792800.

23. Li W, Liu J, Gong L, Zhou Y, Duan D. Posterior intervertebral space debridement, annular bone grafting and instrumentation for treatment of lumbosacral tuberculosis. BMC Surg 2017;17:124.

24. Nas K, Kemaloglu MS, Cevik R, et al. The results of rehabilitation on motor and functional improvement of the spinal tuberculosis. Joint Bone Spine 2004;71:312-6.
25. Omran K, Abdel-Fattah AS, Othman AMA, et al. Lateral extracavitary approach versus posterior extensive circumferential decompression in the treatment of complicated thoracic and lumbar tuberculous spondylitis. Clin Spine Surg 2017;30:E1211-9.

26. Vervoordeldonk JJ, Post MW, New P, Clin Epi M, van Asbeck FW. Rehabilitation of patients with nontraumatic spinal cord injury in the Netherlands: etiology, length of stay, and functional outcome. Top Spinal Cord Inj Rehabil 2013;19:195-201.

27. Vianin M. Psychometric properties and clinical usefulness of the Oswestry Disability Index. J Chiropr Med 2008;7:161-3.

28. Mulcahey MJ, Thielen CC, Sadowsky C, et al. Despite limitations in content range, the SCIM-III is reproducible and a valid indicator of physical function in youths with spinal cord injury and dysfunction. Spinal Cord 2018;56:332-40.

29. Zhu G, Jiang LY, Yi Z, et al. Sacroiliac joint tuberculosis: surgical management by posterior open-window focal debridement and joint fusion. BMC Musculoskelet Disord 2017;18:504.

30. Nas K, Karakoc M, Aydin A, Ones K. Rehabilitation in spinal infection diseases. World J Orthop 2015;6:17.

31. Rabchevsky AG, Kitzman PH. Latest approaches for the treatment of spasticity and autonomic dysreflexia in chronic spinal cord injury. Neurotherapeutics 2011;8:274-82.

32. Engkasan JP, Sudin SS. Neurogenic bowel management after spinal cord injury: Malaysian experience. J Rehabil Med 2013;45:141-4.

33. Taweel WA, Seyam R. Neurogenic bladder in spinal cord injury patients. Res Rep Urol 2015;7:85-99. 